\title{
Mehr Wirtschaftlichkeit und Mobilität beim Blutzuckermessen
}

Das nur $4 \mathrm{~cm}$ kleine Messgerät STADA Gluco Result To Go Plus bietet DiabetesPatienten mehr Mobilität bei hoher Funktionalität - für ein aktives Leben. Das kleinste Blutzuckermessgerät der Welt und sein großer Bruder, das bewährte STADA Gluco Result, verwenden die gleichen STADA Gluco Result Blutzuckerteststreifen. Diese Teststreifen gehören zur günstigeren Preisgruppe nach Ziffer II.1 des Vdek-DAV-Arzneiversorgungsvertrages (Gruppe B) und sind in vielen Fällen wirtschaftlich. Zurzeit bestehen Rabattverträge mit 10 großen Krankenkassen.

Mit der Verschreibung der STADA Gluco Result Teststreifen leistet der Arzt einen aktiven Beitrag zur wirtschaftlichen Verordnung und Kostendämpfung. Der Diabetes-Patient wird durch die ärztliche Empfehlung bzw. Erhalt des kompakten To Go Plus Geräts mit vielen Vorteilen motiviert und in seinem aktiven Lebensstil unterstützt. Das fördert nicht nur die Arzt-Patienten-Bindung, sondern kann auch Typ-2-Diabetiker dazu anhalten, regelmäßig selbst zu messen.

\section{Messkomfort und Genauigkeit}

Das nur walnussgroße STADA Gluco Result To Go Plus hat viele Vorteile:

- Das Messgerät lässt sich auf dem Deckel der Teststreifendose aufschrauben und die Stechhilfe wird mit einer Mobilmanschette ebenfalls an der Dose befestigt. Dieses kleine Set passt in jede Hand-, Jacken- oder Hosentasche und kann einfach einhändig bedient werden.

- Das Display ist gut lesbar. Die Messergebnisse werden mit Datum und Uhrzeit angezeigt. Im Speicher können neben 500 Messwerten auch die Durchschnittswerte von 7, 14 und 30 Tagen eingesehen werden. Das Gerät hat zusätzlich einen USB-Anschluss, sodass der Arzt die Selbstmessungen des Patienten in das Diabetes-Management mit einbeziehen kann.
- Die Messungen sind präzise und erfüllen die Qualitätsrichtlinien nach DIN ISO 15197:2013

- Testgeräte, Zubehör und ServiceMaterialien kann der Arzt kostenfrei anfordern. Es ist jeweils eine GeräteVersion mit mg/dl- oder $\mathrm{mmol} / \mathrm{l}$ Anzeige erhältlich.

- Das Messgerät für unterwegs lässt sich gut mit dem Heimgerät STADA Gluco Result kombinieren, das von Stiftung Warentest als bestes B-Gerät in der Unterkategorie „Genauigkeit der Messung“ mit der Note SEHR GUT $(1,4)$ ausgezeichnet wurde [1]. So kann der Patient Mobilität mit Komfort (Testerinnerungen, Ketonkörperwarnung etc.) verbinden.

\section{Literatur \\ 1 Magazin „Stiftung Warentest Test“ Ausgabe $07 / 2012$}

Pressemitteilung STADA, Bad Vilbel

\section{Individuelles $\mathrm{HbA}_{1 c}$-Ziel ohne Hypoglykämien erreichen!}

Erster Schritt beim Diabetes-Management sollte bei jedem Patienten die Klärung des individuellen Therapieziels sein, betonte Dr. Martina Lange, niedergelassene Diabetologin in Rheinbach, beim Deutschen Diabeteskongress in Berlin. Dann geht es darum, dieses Ziel ohne Hypoglykämien zu erreichen.

Abhängig von Alter, Gesundheit und körperlicher Fitness variiert der $\mathrm{HbA}_{1 \mathrm{c}}{ }^{-}$ Zielwert von $<6,5 \%$ bei jungen gesunden Diabetikern bis $<7,5 \%$ bei älteren multimorbiden Patienten, erinnerte Lange. Medikament der ersten Wahl - zusätzlich zu Ernährungsumstellung und Bewegung ist Metformin. Reicht es nicht aus, stehen gut geeignete neue orale Antidiabetika als Kombinationspartner zur Verfügung. Als Vorteile von DPP-4- und SGLT-2-Hemmern hob Lange das geringe Hypoglykämierisiko und die gewichtsneutrale bzw. -reduzierende Wirkung hervor. DPP-4Hemmer sind zudem in allen Stadien einer Niereninsuffizienz zugelassen.
Die Insulintherapie ist im Management von Typ-2-Diabetikern zwar etwas nach hinten gerückt, sollte aber nicht zu lange hinausgezögert werden. „Wir kommen am Insulin bei Typ-2-Diabetikern nicht vorbei“, betonte Dr. Andreas Lueg vom Diabeteszentrum Hameln. Besonders sinnvoll sei ein frühzeitiger Insulineinsatz bei hohen NüchternBlutzuckerwerten, zur Vermeidung von oralen Kombinationstherapien und von Polypharmakotherapie, bei Medikamentenunverträglichkeit und bei niereninsuffizienten Patienten. Auch bei Patienten mit Blutzuckerentgleisungen ist die Insulintherapie eine wichtige Option und könne hier eventuell interventionell erfolgen.

Die von vielen Patienten befürchtete Gewichtszunahme könne durch umfassende Ernährungsberatung verringert werden, sagte Lueg. Zudem falle bei einer Kombinationstherapie mit oralen Antidiabetika die Gewichtszunahme unter Insulin in der Regel deutlich geringer aus, genauso wie das Risiko für Hypoglykämien. Zur Erhöhung der Therapiesicherheit sollten die Patienten regelmäßig geschult und ins korrekte Messen und Spritzen eingewiesen werden, so der Diabetologe weiter. Zudem sollten auch die Spritzstellen immer mal wieder angeschaut werden. Insbesondere bei Patienten mit hohem Insulinbedarf seien dabei nicht selten große Depotbildungen von Insulin festzustellen, die bei Freisetzungen natürlich zu Unterzuckerungen führen würden. Eine Hilfe können hier hochkonzentrierte Insuline wie Insulin lispro 200 (Liprolog ${ }^{\circledR}$ 200) sein, das im Vergleich zu üblichen schnell wirksamen Insulinen im halben Volumen gespritzt werden kann.

Roland Fath, Hamburg

Quelle: Symposium „Polymorbidität - eine Frage der richtigen Einstellung", am 4. Mai 2016, Berlin, im Rahmen der 51. Jahrestagung der Deutschen Diabetes-Gesellschaft.

Veranstalter: Berlin-Chemie 